\title{
Financial implications of a hospital early mobility program
}

\author{
K Bognar ${ }^{1}$, JW Chou' ${ }^{1}$ D McCoy ${ }^{2 *}$, AL Sexton Ward ${ }^{1}$, J Hester ${ }^{3}$, P Guin ${ }^{3}$, AB Jena ${ }^{4}$ \\ From ESICM LIVES 2015 \\ Berlin, Germany. 3-7 October 2015
}

\section{Introduction}

Decades of advancements in critical care medicine have led to dramatic mortality and morbidity reductions in intensive care units (ICU). Unfortunately, significant advances in neuromuscular and neurocognitive functional recovery have lagged behind [1], despite broad evidence demonstrating that patients who survive critical care admissions may have long-term function declines, and increased long-term morbidity and mortality [2].

Early mobility programs (EMP) have been shown to improve patient clinical outcomes; however, a lack of evidence associated with financial outcomes has served as a barrier to investment for program implementation [3].

\section{Objective}

To evaluate the financial benefits/challenges associated with the published clinical outcomes of EMPs in the US ICU environment.

\section{Methods}

Using literature based clinical outcome estimates of EMPs for ICU patients, we developed a financial impact model and simulated the impact of introducing an EMP in an ICU on costs to hospitals, third-party payers, and capitated healthcare delivery systems. The model includes the following key variables: annual number of ICU admissions by ventilation status and insurance type; improvements in clinical and administrative outcomes e.g. length of stay (LOS), days on ventilator, and rate of hospital readmission; cost savings associated with the clinical and administrative outcomes; and start-up and operating costs.

\section{Results}

The total net present value over a seven-year time horizon of an EMP for a US hospital with 1000 yearly ICU

\footnotetext{
${ }^{2}$ Hill-Rom, Chicago, United States
}

Full list of author information is available at the end of the article admissions exceeds $\$ 2.3 \mathrm{~m}$. The yearly cost-of-care savings generated by reducing ICU and Non-ICU for both ventilated and non-ventilated patients and the number of days on ventilation for a hospital is approximately $\$ 927,000$. The impact of EMPs on hospital readmissions generates an additional $\$ 93,000$ annual savings by reducing US hospital readmission penalties. The model also suggests that EMPs generate even greater value for third-party payers and capitated health systems.

\section{Conclusions}

EMPs may generate substantial clinical improvements in ICU patients and reduce costs to hospitals, payers, and capitated health care delivery systems. Even when the expected clinical effectiveness of an EMP is reduced by $20 \%$ the estimated net present value was positive by the second year of the program.

\section{Grant Acknowledgment}

This study was supported by Hill-Rom Corporation.

\section{Authors' details}

${ }^{1}$ Precision Health Economics, Los Angeles, United States. ${ }^{2}$ Hill-Rom, Chicago, United States. ${ }^{3}$ University of Florida, Gainesville, United States. ${ }^{4}$ Harvard Medical School, Boston, United States.

Published: 1 October 2015

\section{References}

1. Jackson JC, et al: Improving patient care through the prism of psychology: Application of Maslow's hierarchy to sedation, delirium, and early mobility in the intensive care unit. Journal of Critical Care 2014, 29(3):438-444.

2. Desai SV, Law TJ, Needham DM: Long-term complications of critical care. Critical care medicine 2011, 39(2):371-9.

3. Lord RK, et al: ICU early physical rehabilitation programs: financial modeling of cost savings. Critical care medicine 2013, 41(3):717-24.

doi:10.1186/2197-425X-3-S1-A758

Cite this article as: Bognar et al:: Financial implications of a hospital early mobility program. Intensive Care Medicine Experimental 2015 3(Suppl 1):A758. (c) 2015 Bognar et al.; This is an Open Access article distributed under the terms of the Creative Commons Attribution License (http:// creativecommons.org/licenses/by/4.0), which permits unrestricted use, distribution, and reproduction in any medium, provided the original work is properly cited. 